\title{
DA INCONSISTENCIA DA RELAÇÃO ENTRE GRAU DE DUREZA E TEOR DE IÔDO EM ÁGUAS DE ABASTECIMENTO PÚBLICO ${ }^{\circ}$
}

\author{
Francisco Antônio Cardoso * \\ Yaro Ribeiro Gandra ** \\ Germínio Nazário
}

E o bócio endêmico um dos problemas de deficiêneia alimentar que muito tem preocupado as autoridades sanitárias.

Êste interêsse é plenamente justificível tendo em vista além da deformação devida ao aumento da glândula tireóide, ainda, o problema do eretinismo, o da surdo-mudez e o da debilidade mental, com tôdas as suas consequiêneias, relacionados à deficiência iódica.

$\Lambda$ evolução do bócio simples para o bócio nodular tóxico é também um outro ponto a considerar, não só pela gravidade do distúrbio em si, como também pela sua significação cconômica e social, quer por motivar o afastamento do paciente de suas atividades comuns, quer pela necessidade do tratamento cirúrgico, que geralmente se impõe nesses casos.

Embora a etiologia do bócio endêmico não esteja totalmente elucidada é indiscutível que zonas cujas águas e solo são pobres em iôdo constituem aqueJas em que o mal é mais disseminado. É óbvio pois o interêsse que tem a dosagem do iôdo no solo, nas águas e nos alimentos em geral. O conhecimento dessa taxa poderá informar sôbre os limites das zonas bocígenas, além de fornecer elementos científicos importantes para o esclarecimento dos fatores etiológicos responsáveis pela incidência e intensidade da endemia bocígena. O levantamento da riqueza iódica de uma regrião ainda nos proporcionará dados básicos para eampanhas de fornecimento supletivo de iôdo à populaẹão earente.

Em trabalho anterior ${ }^{3}$, tivemos a oportunidade de proceder à determinação do teor de iôdo em águas de abastecimento público dos municípios do Estado de São Paulo, em muitos das quais a endemia bocígena tem sido revelada por alguns pesquisadores. Utilizamos, para isso, a reação entre o cério IV e arsenitos quando eatalizada por iodetos.

Entregue para publicaȩão em 23-10-1957.

- Trabalho da Cadeira de Higiene Alimentar (Prof. Franeiseo Antônio Cardoso) da Faculdade de Higiene e Saúde Públiea da Universidade de São Paulo.

* Professor catedrático.

** Livre docente e assistente da Cadeira.

*** Ex-assistente da Cadeira. 
Vários autores têm sustentado que existe uma relação entre o grau de dureza das águas e o seu teor em iôdo. Este fato vem servindo de justificativa para se substituir a trabalhosa dossagem de iôdo por uma simples determinação da dureza. Com o resultado desta se estimava a riqueza de iôdo da mesma água.

Bado e Trelle 1 , em 61 amostras de água, afirmam que "estabelecem ademais uma relação entre o conteúdo de iôdo e o quociente $\frac{O \mathrm{Na}_{2}}{O \mathrm{Ca}+\mathrm{OMg}}$. Quando êste quociente é menor que 1 as águas carecem de iôdo."

Salas e $\Lambda$ mato ${ }^{\circ}$, analisando 90 amostras de água, dizem que o teor de iôdo "diminue ao aumentar a dureza da água "e que" quando o quociente entre $\frac{\mathrm{Na}+\mathrm{K}}{\mathrm{Ca}+\mathrm{Mg}}$ é menor que 1 , a concentração de iôdo é menor que 5 gamas por litro".

Murray e colahoradores ${ }^{8}$, em 1948, sugerem que a concentração de iôdo em águas moles, embora adequada para prevenir o bóeio, pode não ser suficiente nas águas duras.

Gôngora e colaboradores ${ }^{4}$, em 1949, procedendo a 504 determinações de dureza de águas de consumo público da Colombia e tentando relacioná-las com o bócio endêmico, chegaram à "conclusão de que na Colombia não existe relação bem clara entre o índice de "dureza" das águas de consumo e a percentagem de bócio". Prosseguindo dizem: "Com efeito, existe uma grande maioria de municípios com incidência de bócio simples que ultrapassa de $50 \%$, com águas de consumo perfeitamente brandas. Mas, em troca, nos municípios com águas de consumo catalogadas como duras, ou muito duras, a incidência de bócio simples sempre é maior que $30 \% "$.

Marsano ${ }^{7}$, estudando a dureza e o conteúdo de iôdo das águas de algumas localidades da Argentina, não encontrou relação nítida entre as duas medidas. Exceptuando-se algumas águas, a grande maioria era constituida por águas duras ou muito duras.

Em razão dessa divergência de opiniões existente é que, quando nos propusemos a fazer o levantamento da taxa de iôdo em águas de 369 cidades do Estado de São Paulo ${ }^{3}$ resolvemos, aproveitando o mesmo material da amostra, dosar, concomitantemente, a dureza dessas águas. Visamos com isto perquirir a existência de alguma correlação, positiva ou negativa, entre a dureza das águas e sua riqueza de iôdo; se achada esta relação, iríamos saber se sua perfeição era de tal ordem que nos permitisse estimar o valor de iôdo, quando de posse da dureza.

Material e Técnica - Em 1953, colhemos 381 amostras de águas de abastecimento público de 369 cidades do Estado de São Paulo, sedes de unidades sanitárias do Departamento de Saúde. Aehamos razoável assim proceder, por- 
que conseguiríamos dêste modo uma amostra homogeneamente distribuida por todo o Estado, e, mais ainda, contaríamos, na coleta da amostra, com a ajuda técnica da maioria das unidades sanitárias. Colhemos sempre amostra de águas que efetivamente fôssem consumidas pela maior parte da população. $\boldsymbol{\Lambda}$ coleta destas amostras e as análises foram realizadas no período de Janeiro a Dezembro de 1954.

Em 1955, publicamos as resultados da determinação do teor de iôdo na amostra em questão ${ }^{3}$. Depois de analisar os métodos de dosagem existentes, resolvemos adaptar à dosagem de iôdo na água, a reação cuja cinética fôra estudada por Lein e Sehwartz ${ }^{6}$, ou seja, aquela que ocorre entre o cério IV e os arsenitos, quando catalizada por iodetos. Êste método, adaptado à dosagem de iôdo na água, além de ter sensibilidade capaz de determinar $0,01 \mu \mathrm{g}$ de iodeto, requer pequeno volume de água e é de fácil execução.

Detalhes referentes à coleta das amostras, à preparação dos reagentes e à técnica de dosagem podem ser encontrados em nosso trabalho referido ${ }^{3}$.

A dureza total das águas foi medida pelo processo introduzido por Schwarzenbach ${ }^{10}$, em que ions de cáleio e magnésio são titulados diretamente por solução de sal di-sódico do ácido etilenodiamina-tetracético, que forma quelatos com aqueles íons. A mudança de côr do Negro de Eriocromo T, indica quando aqueles íns se tornam totalmente complexades. $\Lambda$ técnica utilizada foi a de Betz e Noll *, aplicada por Houlihan ", à determinação da dureza total da água.

A fim de estudarmos uma possível correlacãa entre a dureza das águas e o teor de iôdo, a série de valores obtida foi ordenada segundo o gran de dureza.

TABELA I - Resultados da determinação da dureza e da concentração de iôdo das águas dos abastecimentos públicos de 381 localidades do Estado de São Paulo (vide mapas I e II).

CIDADE

Indaiatuba

Conchal

Mandurí $\quad . . \ldots \ldots \ldots \ldots \ldots \ldots \ldots \ldots \ldots \ldots \ldots$

Pôrto Ferreira $\ldots \ldots \ldots \ldots \ldots \ldots \ldots \ldots \ldots$

Pedreira $\quad \ldots \ldots \ldots \ldots \ldots \ldots \ldots \ldots \ldots \ldots \ldots \ldots \ldots$

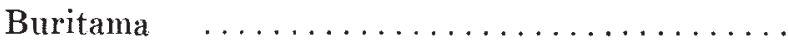

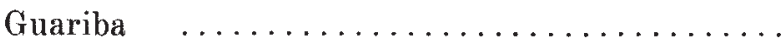

Colina .............................

Itararé

Campos do Jordão

$\begin{array}{cc}\text { Dureza } & \text { Iôdo } \\ \text { p p m } & \mu \mathrm{g} / \text { litro }\end{array}$

$0,8 \quad 0,14$

$0,8 \quad 0,18$

$1,2 \quad 0,22$

$1,6 \quad 0,45$

$2,0 \quad 0,55$

$2,4 \quad 0,73$

$2,4 \quad 0,08$

$2,4 \quad 1,41$

$2,4 \quad 0,09$ 

ARQ. FAC. HIG. SAÚDE PÚB. UNIV. SÃO PAULO, 11 (1) 1957

\begin{tabular}{|c|c|c|}
\hline CIDADE & $\begin{array}{c}\text { Dureza } \\
\text { p p m }\end{array}$ & $\begin{array}{c}\text { Iôdo } \\
\mu \mathrm{g} / \text { litro }\end{array}$ \\
\hline Itirapina & 2,6 & 0,44 \\
\hline Cardoso $\ldots \ldots \ldots \ldots \ldots \ldots \ldots \ldots \ldots$ & 3,2 & 0,08 \\
\hline 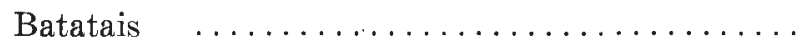 & 3,2 & 0,88 \\
\hline Nuporanga $\quad \ldots \ldots \ldots \ldots \ldots$ & 3,2 & 0,33 \\
\hline Novo Horizonte $\ldots \ldots \ldots \ldots \ldots \ldots \ldots$ & 3,2 & 0,95 \\
\hline 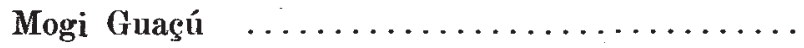 & 3,2 & 0,26 \\
\hline 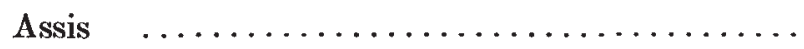 & 3,2 & 0,38 \\
\hline Boituva $\quad \ldots \ldots \ldots \ldots \ldots \ldots \ldots \ldots \ldots \ldots \ldots$ & 3,2 & 0,28 \\
\hline 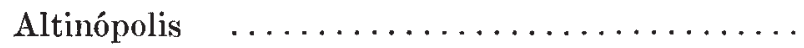 & 4,0 & 0,25 \\
\hline 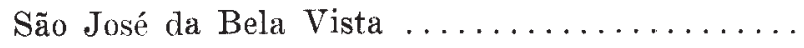 & 4,0 & 0,12 \\
\hline Potirendaba $\quad \ldots \ldots \ldots \ldots \ldots \ldots \ldots \ldots \ldots \ldots \ldots \ldots \ldots \ldots \ldots \ldots \ldots$ & 4,0 & 5,05 \\
\hline Araraquara - Vila Xavier (fonte) ......... & 4,0 & 0,23 \\
\hline Angatuba $\quad \ldots \ldots \ldots \ldots \ldots \ldots \ldots \ldots \ldots \ldots \ldots \ldots \ldots \ldots$ & 4,0 & 0,09 \\
\hline$\ldots \ldots \ldots \ldots$ & 4,0 & 0,82 \\
\hline Ribeirão Branco $\ldots \ldots \ldots \ldots \ldots \ldots \ldots$ & 4,0 & 0,87 \\
\hline Araçoiaba da Serra $\ldots \ldots \ldots \ldots \ldots \ldots \ldots$ & 4,0 & 10,60 \\
\hline$\ldots \ldots \ldots \ldots \ldots \ldots \ldots \ldots \ldots$ & 4,0 & 1,12 \\
\hline 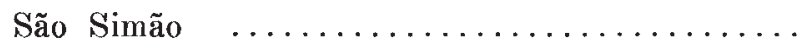 & 4,0 & 0,13 \\
\hline 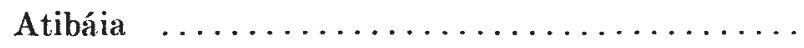 & 4,0 & 0.75 \\
\hline 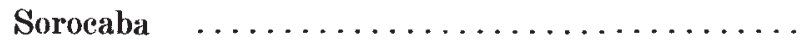 & 4,4 & 7,24 \\
\hline 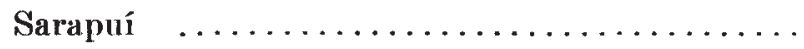 & 4,4 & 0,18 \\
\hline Boa Esperança do Sul $\ldots \ldots \ldots \ldots \ldots \ldots \ldots$ & 4,8 & 0,62 \\
\hline 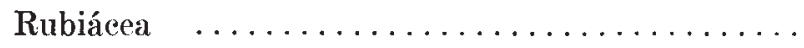 & 4,8 & 0,60 \\
\hline$\ldots \ldots \ldots \ldots \ldots \ldots \ldots \ldots \ldots$ & 4,8 & 0,87 \\
\hline 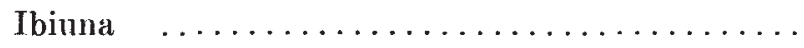 & 4,8 & 0,58 \\
\hline 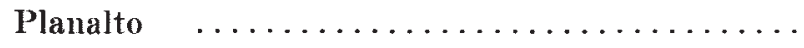 & 5,6 & 0,16 \\
\hline Cerquilho $\quad \ldots \ldots \ldots \ldots \ldots \ldots$ & 5,6 & 0,14 \\
\hline Pedregulho .. & 5,6 & 0,72 \\
\hline 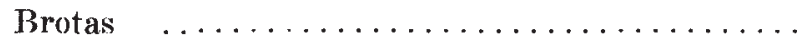 & 5,6 & 0,08 \\
\hline 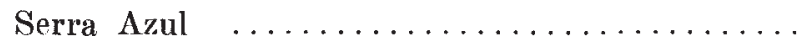 & 5,6 & 0,13 \\
\hline$\ldots \ldots \ldots \ldots \ldots \ldots \ldots \ldots \ldots$ & 5,6 & 2,16 \\
\hline$\ldots \ldots \ldots \ldots \ldots \ldots \ldots \ldots \ldots \ldots \ldots$ & 5,6 & 0,70 \\
\hline$\ldots \ldots \ldots \ldots \ldots \ldots$ & 5,6 & 0,80 \\
\hline Itatinga $\ldots \ldots \ldots \ldots$ & 5,6 & 0.24 \\
\hline Monteiro Lobato & 5,6 & $1, \overline{5} 1$ \\
\hline .Jardinópolis & 6,0 & 0,63 \\
\hline 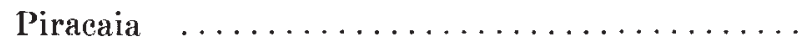 & 6,0 & 2,17 \\
\hline$\ldots \ldots \ldots \ldots \ldots \ldots \ldots \ldots \ldots \ldots$ & 6,4 & 1,96 \\
\hline 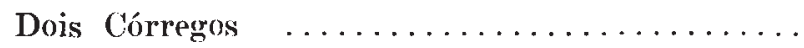 & 6,4 & 2,55 \\
\hline Ribeirão Bonito $\ldots \ldots \ldots \ldots \ldots \ldots \ldots$ & 6,4 & 0,17 \\
\hline$\ldots \ldots \ldots \ldots \ldots \ldots \ldots \ldots \ldots \ldots \ldots$ & 6,4 & 0,36 \\
\hline Santa Cruz das Palmeiras $\ldots \ldots \ldots \ldots \ldots \ldots$ & 6,4 & 0.21 \\
\hline
\end{tabular}


CIDADE

Bilac

Regente Feijó

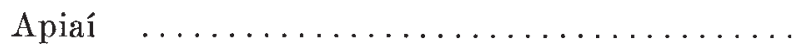

Pindamonhangaba $\ldots \ldots \ldots \ldots \ldots \ldots \ldots \ldots$

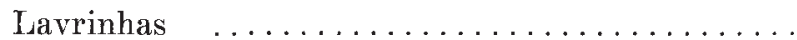

Sales de Oliveira $\ldots \ldots \ldots \ldots \ldots \ldots \ldots \ldots \ldots$

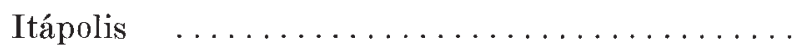

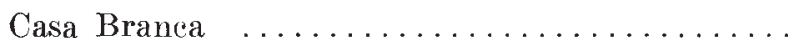

Santa Rita do Passa Quatro ..............

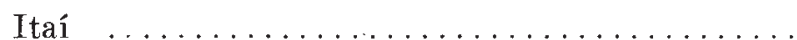

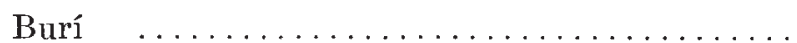

Salesópolis $\ldots \ldots \ldots \ldots \ldots \ldots \ldots \ldots \ldots \ldots$

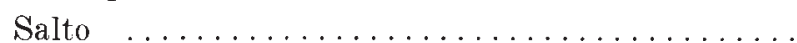

Torrinha $\ldots \ldots \ldots \ldots \ldots \ldots \ldots \ldots \ldots \ldots \ldots$

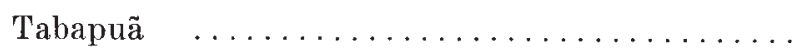

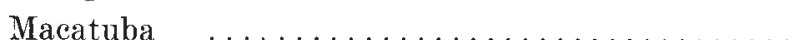

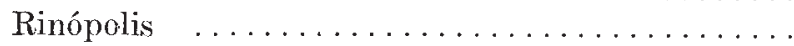

Paranapanema $\ldots \ldots \ldots \ldots \ldots \ldots \ldots \ldots \ldots$

São Paulo - Vila Deodoro ..............

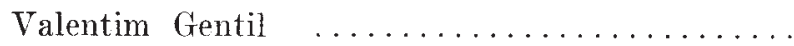

Amparo $\ldots \ldots \ldots \ldots \ldots \ldots \ldots \ldots \ldots \ldots$

Silveiras $\quad \ldots \ldots \ldots \ldots \ldots \ldots \ldots \ldots \ldots \ldots \ldots$

Brodosqui $\ldots \ldots \ldots \ldots \ldots \ldots \ldots \ldots \ldots \ldots \ldots$

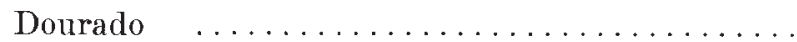

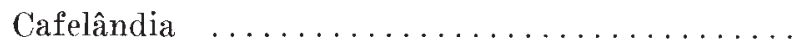

Mineiros do Tietê $\ldots \ldots \ldots \ldots \ldots \ldots \ldots \ldots$

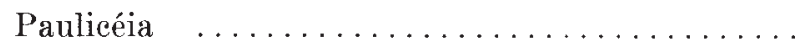

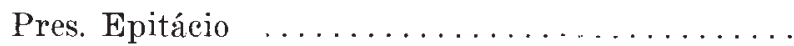

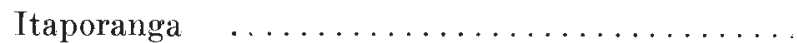

Itapecerica da Serra $\ldots \ldots \ldots \ldots \ldots \ldots \ldots$

Santana do Parnaíba...$\ldots \ldots \ldots \ldots \ldots \ldots$

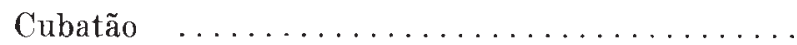

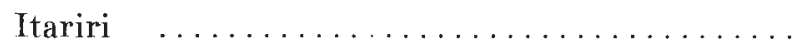

Mogi das Cruzes ...................

Guararema $\ldots \ldots \ldots \ldots \ldots \ldots \ldots \ldots \ldots$

Aparecida do Norte ..................

Lorena $\ldots \ldots \ldots \ldots \ldots \ldots \ldots \ldots \ldots \ldots \ldots \ldots$

Queluz

São Carlos

Timburi

Mairiporã

Jaborandí

$\begin{array}{cc}\text { Dureza } & \text { Iôdo } \\ \text { p p m } & \mu \mathrm{g} / \text { litro }\end{array}$

$6,4 \quad 0,46$

$6,4 \quad 0,56$

$6,4 \quad 0,62$

$6,4 \quad 0,21$.

$6,4 \quad 0,40$

$7,2 \quad 0,83$

$7,2 \quad 0,86$

$7,2 \quad 0,38$

$7,2 \quad 1,23$

$7,2 \quad 0,22$

$7,2 \quad 0,37$

$7,2 \quad 2,37$

$7,2 \quad 1,35$

$8,0 \quad 0,67$

$8,0 \quad \mathbf{1 , 0 6}$

$8,0 \quad 0,42$

$8,0 \quad 1,41$

$8,0 \quad 0,89$

$8,0 \quad 0,45$

$8,0 \quad 0,16$

$8,4 \quad 0,65$

$8,4 \quad 0,63$

$8,8 \quad 0,48$

$8,8 \quad 0,70$

$8,8 \quad 0,93$

$8,8 \quad 0,47$

$8,8 \quad 5,05$

$8,8 \quad 1,23$

$8,8 \quad 0,12$

$8,8 \quad 0,80$

$8,8 \quad 0,61$

$8,8 \quad 0,45$

$8,8 \quad 1,28$

$8,8 \quad 0,15$

$8,8 \quad 3,31$

$8,8 \quad \mathbf{1}, 63$

$8,8 \quad 0,24$

$8,8 \quad 0,53$

$9,2 \quad 0,76$

$9,2 \quad 0,34$

$9,2 \quad 1,85$

$9,6 \quad 0,05$ 
CIDADE

Tabatinga

Lindóia

Jundiaí

Ubirajara

Jambeiro

Natividade da Serra

Cachoeira Paulista

Bananal

Guaira

Descalvado

Pinhal

Cordeirópolis

Echaporã

Cerqueira César

Bofete

São Vicente

Paraibuna

Caraguatatuba

Cruzeiro

Ubatuba

Bocaina

Jarinú

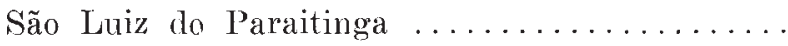

Macaubal

Socorro

Franco Rocha

Martinópolis

Rancharia

Itapetininga

Joanópolis

Santa Isabel

Tatuí

Igarapava

São Joaquim da Barra

São Manoel

Terra Roxa

Pitangueiras

Limeira

Serra Negra

Cananéia

Taubaté

Ilha Bela

\section{Dureza}

p p m

9,6

9,6

9.6

9.6

9,6

9,6

9,6

9.6

10,0

10,0

10,4

10,4

10,4

10,4

10,4

10,4

10,4

10,4

10,4

10,4

10,8

10,8

10.8

11,2

11.2

11,2

11,2

11,2

11,2

11,2

11,2

11,2

11,2

11,6

12,0

12,0

12,0

12,0

12,0

12,0

12,0

12,0
Iôdo $\mu \mathrm{g} /$ litro

0,16

0,81

0,74

0,94

1,88

2,47

0,23

3,40

0,19

4,10

0.56

6,00

3,75

0,47

0,35

1,05

0,71

2,83

0,18

8,64

0,93

0,89

1,87

4,45

0,56

0,51

0,58

0,33

0,45

0,09

4,29

1,03

0,72

0,19

0,62

0,15

1,87

1,20

0,28

1,60

1,11

3,60 


\begin{tabular}{|c|c|c|}
\hline CIIDADE & $\begin{array}{c}\text { Dureza } \\
\text { p pm }\end{array}$ & $\begin{array}{c}\text { Iôdo } \\
\mu \mathrm{g} / \text { /litro }\end{array}$ \\
\hline Guaratinguetá & 12,0 & 0,22 \\
\hline$\ldots \ldots \ldots \ldots \ldots \ldots \ldots \ldots \ldots \ldots$ & 12,0 & 0,12 \\
\hline São José do Barreiro . . . . . . . . . . . . . & 12,0 & 0,16 \\
\hline$\ldots \ldots \ldots \ldots \ldots \ldots \ldots \ldots \ldots \ldots$ & 12,4 & 0,27 \\
\hline Lençóis Paulista & 12,4 & 0,77 \\
\hline São Paulo - Mooca ...... & 12,8 & 0,53 \\
\hline São Sebastião da Grama $\ldots \ldots \ldots \ldots \ldots \ldots$ & 12,8 & 2,55 \\
\hline 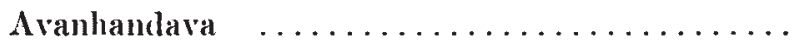 & 12,8 & 0,30 \\
\hline Capivarí $\ldots \ldots \ldots \ldots \ldots \ldots \ldots \ldots \ldots$ & 12,8 & 1,37 \\
\hline Monte Aleyre do Sul .... & 12,8 & $0,7 \bar{j}$ \\
\hline Cândido Mota $\ldots . . .$. & 12,8 & 0,60 \\
\hline 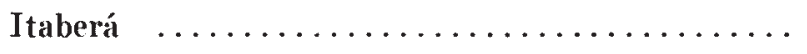 & 12,8 & 0,41 \\
\hline Nazaré Paulista . . . . . . . . . & 12,8 & 0,18 \\
\hline Pilar do Sul . . . . . . . . . . . . . . . & 12,8 & 0,84 \\
\hline Iguape $\quad \ldots \ldots \ldots \ldots \ldots \ldots \ldots \ldots \ldots \ldots$ & 12,8 & 1,89 \\
\hline Redenção da Serra $\ldots \ldots \ldots \ldots \ldots \ldots \ldots$ & 12,8 & 1,19 \\
\hline 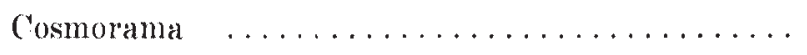 & 13,2 & 0,53 \\
\hline Jaboticabal $\quad \ldots \ldots \ldots \ldots \ldots \ldots \ldots \ldots$ & 13,6 & 0,79 \\
\hline Guararapes $\quad \ldots \ldots \ldots \ldots \ldots \ldots \ldots \ldots \ldots \ldots$ & 13,6 & 1.63 \\
\hline Itanhaem $\quad \ldots \ldots \ldots \ldots \ldots \ldots \ldots \ldots \ldots$ & 13.6 & $0,7.5$ \\
\hline 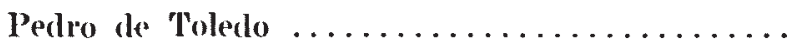 & 13.6 & 2,12 \\
\hline 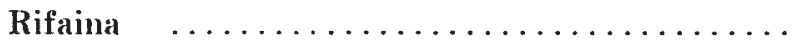 & 13.6 & 0.64 \\
\hline 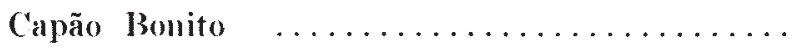 & 14,4 & 0,66 \\
\hline 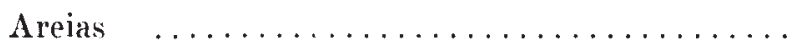 & 14,4 & 1,03 \\
\hline 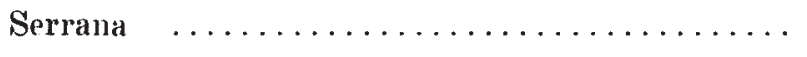 & 15,0 & 0,00 \\
\hline 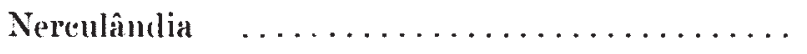 & 15,2 & 0,90 \\
\hline Santa Branca $\ldots \ldots \ldots \ldots \ldots \ldots \ldots \ldots$ & 15,2 & 1,18 \\
\hline São Paulo - Penha $\ldots \ldots \ldots \ldots \ldots \ldots$ & 15,2 & 0,47 \\
\hline Itapuí $\ldots \ldots \ldots \ldots \ldots \ldots \ldots \ldots \ldots \ldots$ & 15,2 & 6,40 \\
\hline General Salgrado $\ldots \ldots \ldots \ldots \ldots \ldots \ldots \ldots$ & 16,0 & 8,45 \\
\hline 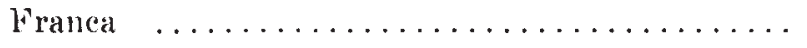 & 16.0 & 0,19 \\
\hline 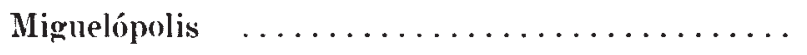 & 16.0 & 0,34 \\
\hline Pederneiras ... & 16,0 & 3,78 \\
\hline 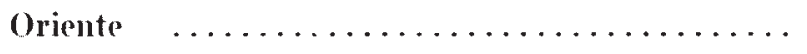 & 16,0 & 2,25 \\
\hline São Caetano do Sul ... & 16,0 & 3,17 \\
\hline 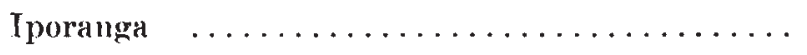 & 16,0 & 1,33 \\
\hline São Paulo - Vila Mariana . . . . . . . . . . . . & 16,0 & 3,65 \\
\hline$\ldots \ldots \ldots \ldots \ldots \ldots \ldots \ldots$ & 16,1 & 0,48 \\
\hline Póá $\quad \ldots \ldots$. & 16,6 & 1,39 \\
\hline Guarantã & 16,8 & 0,25 \\
\hline 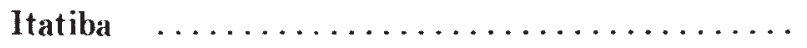 & 16,8 & 1,71 \\
\hline
\end{tabular}




\section{CIDADE}

São Pedro

Chavantes

São Bento do Sapucaí

Guarujá

Viradouro

Taquaritinga

Coroados

Avaí

Parapuã

Mogi Mirim

Santa Cruz do Rio Pardo

Laranjal Paulista

Itirapuã

Corumbataí

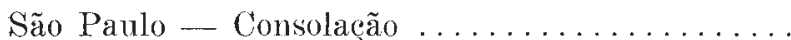

Américo de Campos $\ldots \ldots \ldots \ldots \ldots \ldots \ldots \ldots$

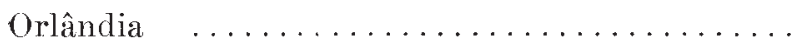

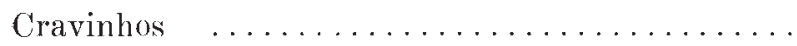

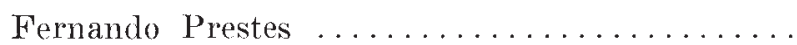

Ituverava $\ldots \ldots \ldots \ldots \ldots \ldots \ldots \ldots \ldots \ldots \ldots$

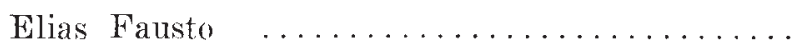

Santos

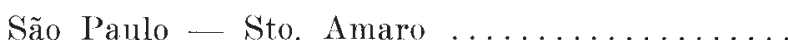

Americana

Vinhedo

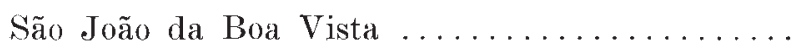

Cotia

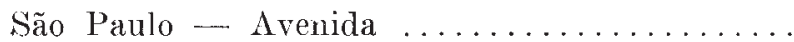

São Paulo Vila América $\ldots \ldots \ldots \ldots \ldots \ldots \ldots \ldots$

Ibirá

Bastos

Campinas

Araras

Ipauçú

Araraquara (reprêsa artificial)

Taquarituba

Itú

Jacaré́

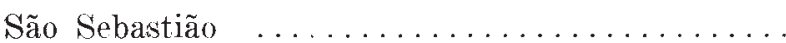

Anhembí

Pôrto Feliz

\section{Dureza Iôdo \\ p p m $\quad \mu g /$ litro}

$\begin{array}{ll}16,8 & 1,63 \\ 16,8 & 1,23 \\ 16,8 & 0,39 \\ 17,2 & 3,02 \\ 17,6 & 0,58 \\ 17,6 & 0,91\end{array}$

$17,6 \quad 7,20$

$17,6 \quad 0,25$

$17,6 \quad 0,20$

$17,6 \quad 1,07$

$17,6 \quad 0,29$

$17,6 \quad 2,49$

$18,0 \quad 0,88$

$18,0 \quad 1,43$

$18,4 \quad 2,09$

$18,4 \quad 0,65$

$18,4 \quad 3,20$

$18,4 \quad 1,31$

$18,4 \quad 0,24$

$18,8 \quad 1,32$

$18,8 \quad 0,15$

$18,8 \quad 0,20$

$19,2 \quad 2,20$

$19,2 \quad 2,95$

$19,2 \quad 1,59$

$19,2 \quad 1,57$

$19,2 \quad 5,04$

$20,0 \quad 4,27$

$20,0 \quad 1,60$

$20,0 \quad 1,98$

$20,0 \quad 3,35$

$20,0 \quad 1,38$

$20,0 \quad 3,32$

$20,0 \quad 0,73$

$20,0 \quad 0,39$

$20.0 \quad 1,18$

$20,0 \quad 0,56$

$20,0 \quad 0,77$

$20,0 \quad 4,65$

$20,4 \quad 2,75$

$20,4 \quad 1,47$ 
CARDOSO, F. A. \& COLS. - DA INCONSISTÊNCIA DA REIJAÇÃO...

CIDADE

Miracatu

São José dos Campos

Nhandeara

Tupã

São Paılo - Água Branca

Pongaí

Bragança Paulista $\ldots \ldots \ldots \ldots \ldots \ldots \ldots$

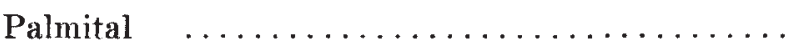

São Miguel Arcanjo $\ldots \ldots \ldots \ldots \ldots \ldots \ldots \ldots$

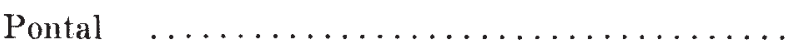

Santa Barbara do Oeste ................

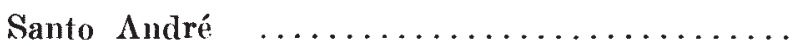

Campos Novos Paulista..$\ldots \ldots \ldots \ldots \ldots \ldots$

Botucatı

São Roque

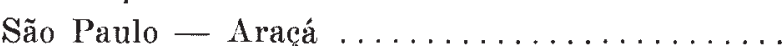

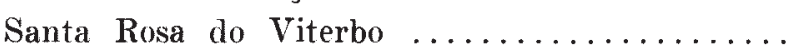

Tremembé

Jacupiranga

São Paulo - Santaula

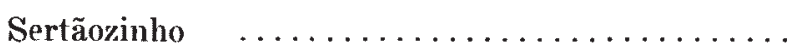

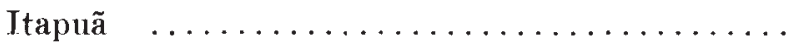

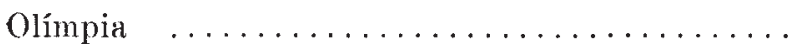

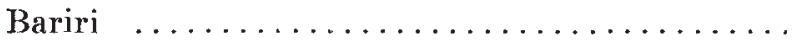

Mocóca $\quad \ldots \ldots \ldots \ldots \ldots \ldots \ldots \ldots \ldots \ldots$

Júlio Mesquita $\ldots \ldots \ldots \ldots \ldots \ldots \ldots \ldots \ldots$

Itapira $\quad \ldots \ldots \ldots \ldots \ldots \ldots \ldots \ldots \ldots$

Álvares Machado $\ldots \ldots \ldots \ldots \ldots \ldots \ldots . . . \ldots$.

São Bernardo do Campo ...............

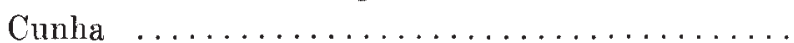

São Paulo - Freguesia do $0 \ldots \ldots \ldots \ldots \ldots$

Cirupês

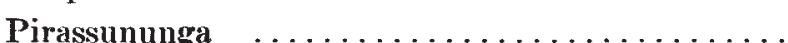

São José do Rio Pardo $\ldots \ldots \ldots \ldots \ldots \ldots \ldots \ldots$

Lins

Agudos

Santa Bárbara do Rio Pardo

Tambaú

Juquiá

Suzano

\section{Dureza Iôdo \\ p p m $\quad \mu \mathrm{g} /$ litro}

$20,8 \quad 4,71$

$20,8 \quad 0,42$

$20,8 \quad 3,33$

$21,2 \quad 3,50$

$21,6 \quad 18,10$

$21,6 \quad 0,75$

$21,6 \quad 1,89$

$21,6 \quad 0,88$

$21,6 \quad 0,69$

$21,6 \quad 0,35$

$22,4 \quad 0,65$

$22,4 \quad 1,20$

$22,4 \quad 1,39$

$22,4 \quad 0,95$

$22,4 \quad 0,54$

$22,4 \quad 3,55$

$23,2 \quad 17,00$

$23,2 \quad 0,92$

$23,2 \quad 7,25$

$23,6 \quad 5,94$

$24,0 \quad 1,10$

$24,0 \quad 0,62$

$24,0 \quad 0,05$

$24,0 \quad 2,20$

$24,0 \quad 6,75$

$24,0 \quad 1,29$

$24,0 \quad 0,26$

$24,0 \quad 1,52$

24,0 0,16

$24,0 \quad 1,72$

$24,4 \quad 2,77$

$24,8 \quad 16,16$

$24,8 \quad 0,91$

$25,6 \quad 1,95$

$25,6 \quad 0,93$

$25,610,70$

$25,6 \quad 0,46$

$25,6 \quad 0,81$

$26,0 \quad 2,83$

$26,0 \quad 1,22$

$26,0 \quad 1,05$ 
(1) 1957

\begin{tabular}{|c|c|c|}
\hline CIDADE & $\begin{array}{c}\text { Dureza } \\
\text { p p m }\end{array}$ & $\begin{array}{c}\text { Iôdo } \\
\mu \mathrm{g} / \text { litro }\end{array}$ \\
\hline Analândia & 26,4 & 0,77 \\
\hline 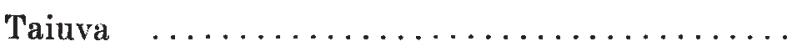 & 26,4 & 0,39 \\
\hline Santa Adélia $\ldots \ldots \ldots \ldots \ldots \ldots \ldots \ldots$ & 26,4 & 1,25 \\
\hline Barra Bonita $\ldots \ldots \ldots \ldots \ldots \ldots \ldots$ & 26,4 & 0,56 \\
\hline Irapuã $\quad \ldots \ldots \ldots \ldots \ldots \ldots \ldots \ldots \ldots$ & 26,4 & 1,05 \\
\hline Patrocínio Paulista $\ldots \ldots \ldots \ldots \ldots \ldots \ldots \ldots$ & 27,2 & 0,82 \\
\hline Santo Antônio da Alegria .............. & 27,2 & 0,74 \\
\hline Ibirarema $\quad \ldots \ldots \ldots \ldots \ldots \ldots \ldots \ldots$ & 27,6 & 0,57 \\
\hline$\ldots \ldots \ldots \ldots \ldots \ldots \ldots \ldots \ldots$ & 28,0 & 0,96 \\
\hline$\ldots \ldots \ldots \ldots \ldots \ldots$ & 28,0 & 0,35 \\
\hline 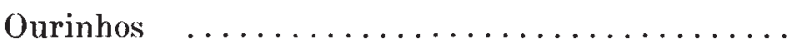 & 28,0 & 1,75 \\
\hline 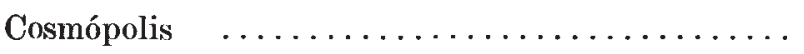 & 29,6 & 0,89 \\
\hline Barretos $\ldots \ldots \ldots \ldots \ldots \ldots \ldots \ldots \ldots \ldots$ & 30,0 & 0,89 \\
\hline$\ldots \ldots \ldots \ldots \ldots \ldots \ldots$ & 30,4 & 2,45 \\
\hline$\ldots \ldots \ldots \ldots \ldots \ldots \ldots$ & 30,4 & 1,43 \\
\hline$\ldots \ldots \ldots \ldots \ldots \ldots$ & 30,4 & 0,71 \\
\hline$\ldots \ldots \ldots \ldots \ldots \ldots \ldots$ & 30,8 & 0,69 \\
\hline 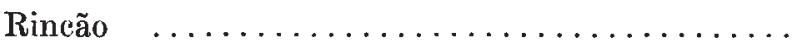 & 31,2 & 1,14 \\
\hline$\ldots \ldots \ldots \ldots \ldots \ldots \ldots$ & 31,2 & 1,44 \\
\hline$\ldots \ldots \ldots \ldots \ldots \ldots \ldots$ & 32,0 & 0,21 \\
\hline$\ldots \ldots \ldots \ldots \ldots \ldots \ldots$ & 32,0 & 1,63 \\
\hline Vargem Grande do Sul ........ & 32,8 & 3,59 \\
\hline$\ldots \ldots \ldots \ldots \ldots \ldots \ldots \ldots \ldots \ldots$ & 33,6 & 5,80 \\
\hline 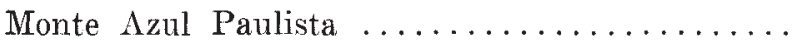 & 33,6 & 1,11 \\
\hline$\ldots \ldots \ldots \ldots \ldots \ldots \ldots \ldots \ldots \ldots$ & 33,6 & 0,80 \\
\hline Caçapava & 34,4 & 1,67 \\
\hline Santa Gertrudes $\ldots$. & 35,2 & 0,72 \\
\hline Águas de São Pedro . & 35,2 & 3,68 \\
\hline Eldorado Paulista $\ldots \ldots \ldots \ldots \ldots \ldots \ldots$ & 36,0 & 1,91 \\
\hline Paulo de Faria .... & 36,0 & 0,49 \\
\hline$\ldots \ldots \ldots \ldots \ldots$ & 36,8 & 2,40 \\
\hline Cabrália Pauljsta & 36,8 & 0,36 \\
\hline 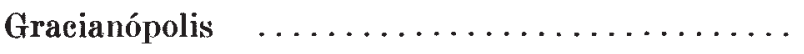 & 36.8 & 0,09 \\
\hline Fernandópolis $\quad \ldots \ldots \ldots \ldots \ldots \ldots \ldots \ldots$ & $: 37,6$ & 0,44 \\
\hline$\ldots \ldots \ldots \ldots \ldots \ldots \ldots \ldots$ & 37,6 & 0,90 \\
\hline$\ldots \ldots \ldots \ldots \ldots \ldots \ldots$ & 38,4 & 19,40 \\
\hline Nova Aliança & 38,4 & 2,52 \\
\hline$\ldots \ldots \ldots \ldots \ldots \ldots$ & 39,2 & 0,10 \\
\hline Morro Agudo $\ldots \ldots \ldots \ldots \ldots \ldots \ldots \ldots$ & 40,0 & 1,86 \\
\hline Penápolis & 40,0 & 0,76 \\
\hline Neves Paulista ............ & 40,4 & 2,98 \\
\hline
\end{tabular}


CIDADE

Tapiratiba

São Pedro do

Cajobi

Bebedouro

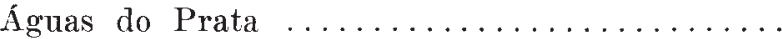

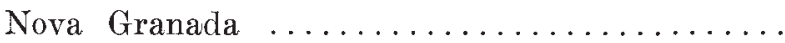

Monte Mór

Paraguaçú Paulista

Itapéva

Catanduva

Junqueirópolis

Oswaldo Cruz

Tietê

Palestina $\ldots \ldots \ldots \ldots \ldots \ldots \ldots \ldots \ldots \ldots \ldots$

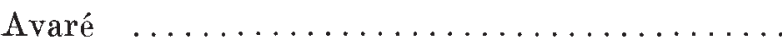

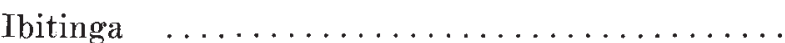

Promissão

Pres. Wenceslau

Monte Aprazível

Pirangi

Alfredo Marcondes

Monte Alto

Lavínia

Ribeira

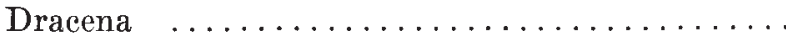

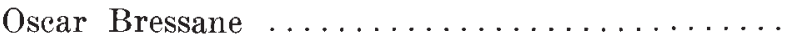

Gália

Barueri

Pindorama $\ldots \ldots \ldots \ldots \ldots \ldots \ldots \ldots \ldots \ldots \ldots$

Tanabi

Matão

Mirandópolis

Fartura

Getulina

Mirassol

Reginópolis

Piratininga

Pereira Barreto

Votuporanga

$\begin{array}{cc}\text { Dureza } & \text { Iôdo } \\ \text { p p m } & \mu \mathrm{g} / \text { litro }\end{array}$

$40,4 \quad 2,65$

$40,4 \quad 0,85$

$41,6 \quad 0,64$

$42,4 \quad 1,37$

$42,4 \quad 1,00$

$43,2 \quad 0,85$

$44,0 \quad 0,65$

$44,0 \quad 4,75$

$44,0 \quad 0,66$

$44,0 \quad 0,38$

$45,6 \quad 2,59$

$45,6 \quad 0,67$

$46,0 \quad 2,03$

$46,0 \quad 1,11$

$47,2 \quad 1,18$

$47,2 \quad 0,34$

$48,8 \quad 1,71$

$48,8 \quad 3,48$

$48,8 \quad 4,85$

$49,6 \quad 1,63$

$49,6 \quad 1,80$

$49,6 \quad 0,70$

$50,4 \quad 0,44$

$50,4 \quad 6,40$

$51,2 \quad 0,33$

$52,0 \quad 0,61$

$52,0 \quad 1,66$

$52,0 \quad 4,75$

$52,8 \quad 1,18$

$53,6 \quad 0,05$

$54,4 \quad 0,30$

$56,0 \quad 0,90$

$57,2 \quad 1,60$

$59,6 \quad 0,19$

$60,0 \quad 4,55$

$60,0 \quad 1,39$

$61,6 \quad 1,62$

$62,0 \quad 1,14$

$64,0 \quad 2,32$

$64,0 \quad 4,85$

$65,6 \quad 1,50$ 


\begin{tabular}{|c|c|c|}
\hline CIDADE & $\begin{array}{c}\text { Dureza } \\
\text { p p m }\end{array}$ & $\begin{array}{c}\text { Iôdo } \\
\mu \mathrm{g} / \text { litro }\end{array}$ \\
\hline Araçatuba $\ldots \ldots \ldots$ & 66,4 & 0,57 \\
\hline 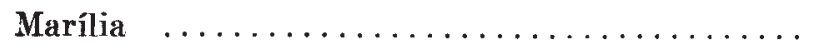 & 66,4 & 6,80 \\
\hline Adamantina $\ldots .$. & 66,4 & 0,82 \\
\hline 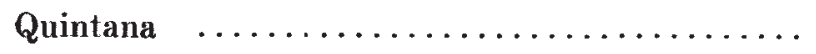 & 67,2 & 1,11 \\
\hline Cabreúva $\quad \ldots \ldots \ldots \ldots \ldots \ldots \ldots \ldots \ldots$ & 68,0 & 4,40 \\
\hline Cedral $\quad \ldots \ldots \ldots \ldots \ldots \ldots \ldots \ldots \ldots \ldots \ldots$ & 68,0 & 0.25 \\
\hline 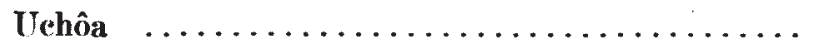 & 68.4 & 1,21 \\
\hline Bernardino de Campos $\ldots \ldots \ldots \ldots \ldots \ldots \ldots \ldots$ & 69,6 & 1,05 \\
\hline$\ldots \ldots \ldots$ & 72,0 & 0,32 \\
\hline Salto Grande $\ldots \ldots \ldots \ldots \ldots \ldots \ldots \ldots$ & 72,0 & 1,02 \\
\hline Vera Cruz $\ldots \ldots \ldots \ldots \ldots \ldots \ldots \ldots \ldots$ & 72,8 & 1,70 \\
\hline Pres. Alves $\ldots \ldots \ldots \ldots \ldots \ldots \ldots \ldots$ & 72,8 & 0,46 \\
\hline 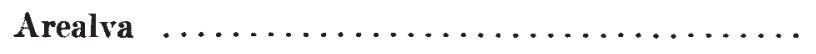 & $\mathbf{7 4 . 4}$ & $\mathbf{0 , 5 5}$ \\
\hline Pres. Bernardes .... & 79,2 & 1.78 \\
\hline Estrêla do Oeste .... & 80,4 & 0,02 \\
\hline 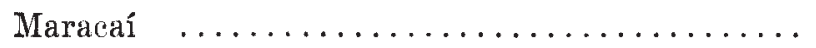 & 81,6 & 0,70 \\
\hline Jales $\quad \ldots \ldots \ldots \ldots \ldots \ldots \ldots \ldots \ldots \ldots \ldots$ & 83,2 & 0,35 \\
\hline Duartina $\ldots \ldots \ldots \ldots \ldots \ldots \ldots \ldots \ldots$ & 84,0 & 0,37 \\
\hline Conchas $\quad \ldots \ldots \ldots \ldots \ldots \ldots \ldots \ldots \ldots \ldots \ldots \ldots \ldots \ldots \ldots \ldots \ldots$ & 85.6 & 0.76 \\
\hline 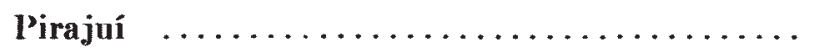 & 86,4 & 0,80 \\
\hline 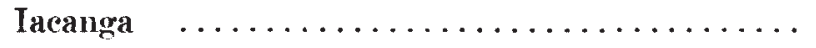 & 91,2 & 1,81 \\
\hline 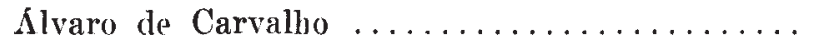 & 91,2 & 0,32 \\
\hline 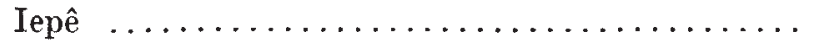 & 91,6 & 1,62 \\
\hline Santo $\Lambda$ nastácio . . . . . . . . . . . . . . & 93,2 & 21,15 \\
\hline Pirapòzinho $\ldots \ldots \ldots \ldots \ldots \ldots \ldots \ldots \ldots \ldots \ldots \ldots \ldots \ldots$ & 93,6 & 0,97 \\
\hline Banru $\ldots \ldots \ldots \ldots \ldots \ldots \ldots \ldots \ldots \ldots \ldots \ldots \ldots$ & 96.0 & 4.42 \\
\hline José Bonifácio $\ldots \ldots \ldots \ldots \ldots \ldots \ldots \ldots \ldots \ldots$ & 97,6 & 2,64 \\
\hline$\ldots \ldots \ldots \ldots \ldots \ldots \ldots \ldots \ldots \ldots$ & 100,8 & 4,80 \\
\hline Pres. Prudente $\ldots \ldots \ldots \ldots \ldots \ldots \ldots \ldots \ldots$ & 101,6 & 4,70 \\
\hline 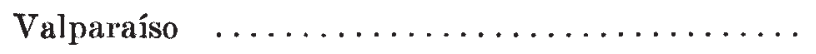 & 108,0 & 0,77 \\
\hline 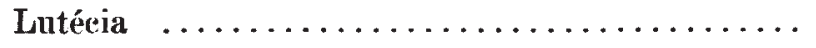 & 116,0 & 9,04 \\
\hline 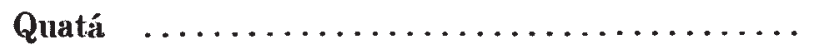 & 129,6 & 1,51 \\
\hline Lucélia $\quad \ldots \ldots \ldots \ldots \ldots \ldots \ldots \ldots \ldots$ & 148,0 & $\mathbf{3 , 5 5}$ \\
\hline 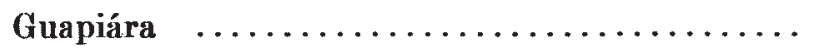 & 152,0 & 1,02 \\
\hline Registro $\ldots \ldots \ldots \ldots$ & 165,6 & 2,10 \\
\hline Flórida Paulista $\ldots \ldots \ldots \ldots \ldots \ldots \ldots \ldots \ldots$ & 185,6 & 4,81 \\
\hline 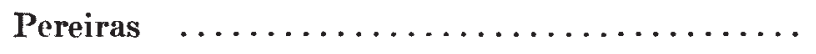 & 216,8 & 6,89 \\
\hline Andradina $\ldots \ldots \ldots \ldots \ldots \ldots \ldots \ldots$ & 244,0 & 1,88 \\
\hline Pacaembu $\quad \ldots \ldots \ldots \ldots \ldots \ldots \ldots \ldots \ldots \ldots \ldots \ldots \ldots \ldots \ldots$ & 259,2 & 1,63 \\
\hline 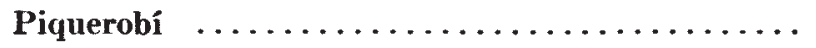 & 288,0 & 1,60 \\
\hline
\end{tabular}




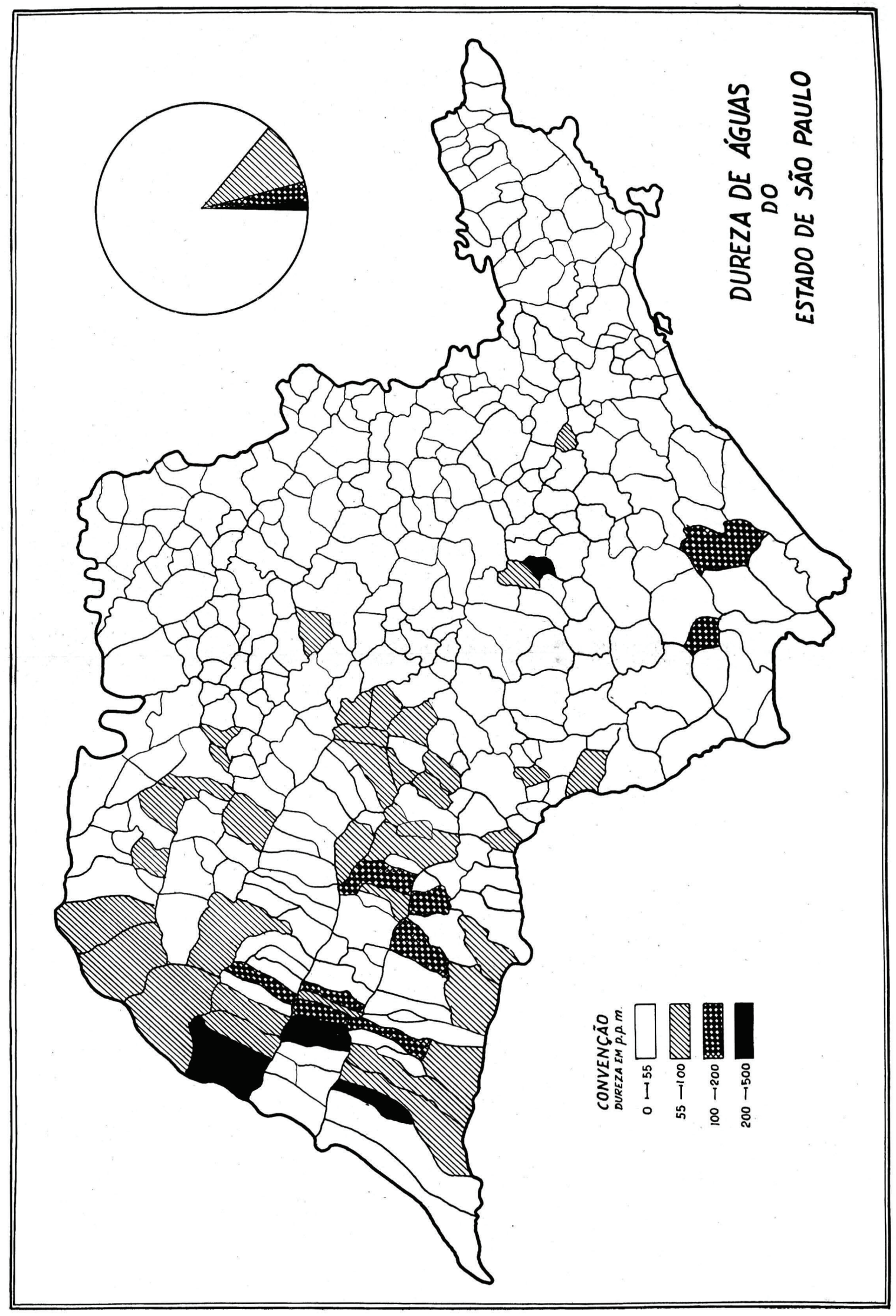




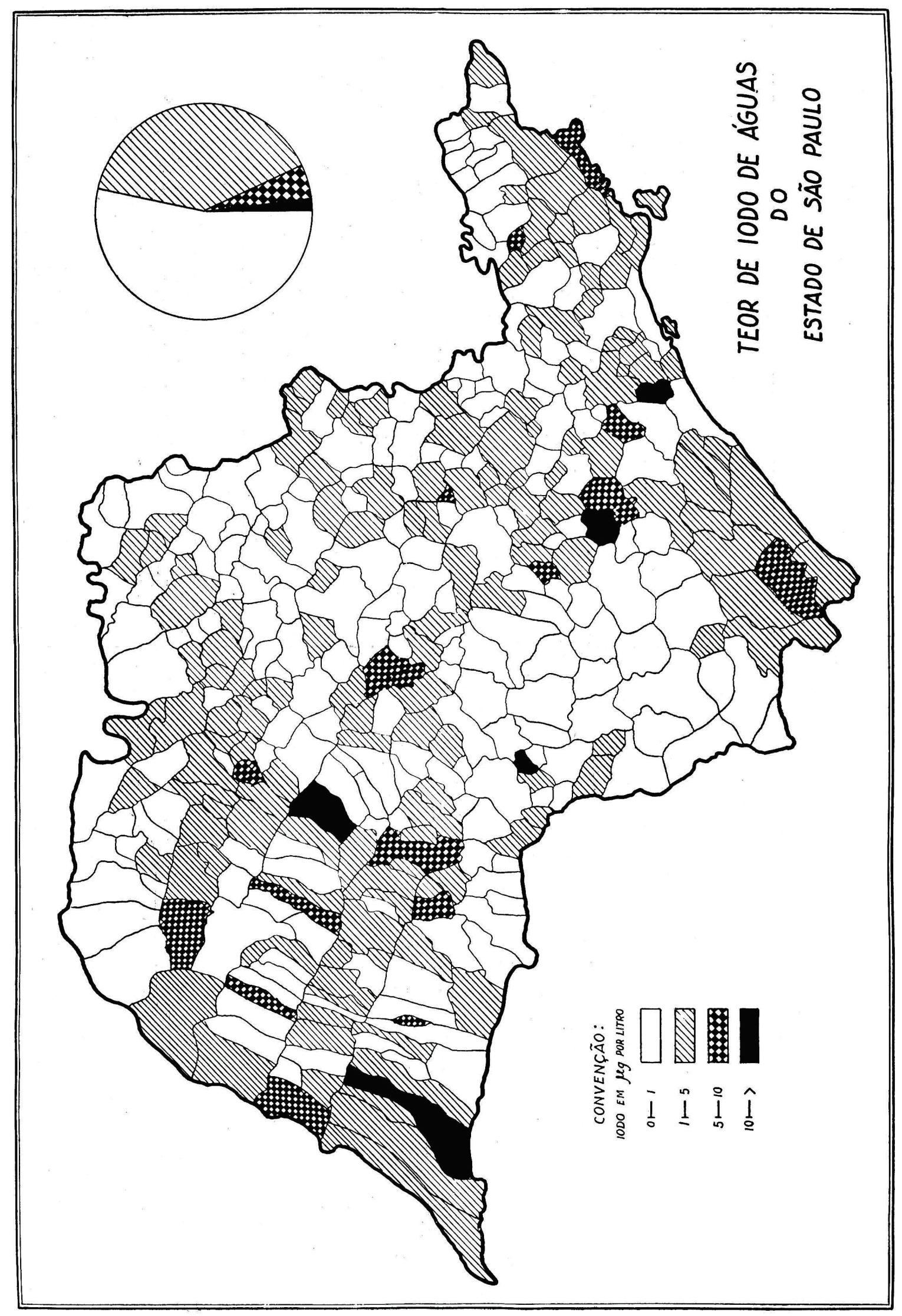


Os resultados da dosagem do iôdo apresentam as seguintes características:

\section{QUADRO I}

\begin{tabular}{|c|c|c|c|c|}
\hline Grupos & $\begin{array}{c}\text { Iôdo em } \mu g \text { por } \\
\text { litro }\end{array}$ & Freqüência & $\begin{array}{l}\text { Percentagem sôbre } \\
\text { o total de casos }\end{array}$ & $\begin{array}{l}\text { Percentagens } \\
\text { acumuladas }\end{array}$ \\
\hline I & $0-1$ & 206 & 54,07 & 54,07 \\
\hline II & $1-5$ & 151 & 39,63 & 93,70 \\
\hline III & $5-10$ & 17 & 4,46 & 98,16 \\
\hline IV & $10 \mathrm{e}+$ & 7 & 1,84 & 100,00 \\
\hline & Total & 381 & 100,00 & \\
\hline
\end{tabular}

Média $=1,73 \mu \mathrm{g}$ por litro

Desvio padrão $=2,526$

Êrro padrão da média $=0,129$

Os resultados da determinação da dureza assiin se distribuem:

\section{QUADRO II}

\begin{tabular}{|c|c|c|c|c|}
\hline Grupos & $\begin{array}{c}\text { Dureza em } \\
\mathrm{p} \mathrm{p} \mathrm{m}\end{array}$ & Freqüência & $\begin{array}{c}\text { Percentagem sôbre } \\
\text { o total de casos }\end{array}$ & $\begin{array}{c}\text { Percentagens } \\
\text { acumuladas }\end{array}$ \\
\hline I & $0-55$ & 322 & 84,52 & 84,52 \\
\hline II & $55-100$ & 46 & 12,07 & 96,59 \\
\hline III & $100-200$ & 9 & 2,36 & 98,95 \\
\hline IV & $200-500$ & 4 & 1,05 & 100,00 \\
\hline & \multirow{2}{*}{ Total } & 381 & 100,00 & \\
\hline
\end{tabular}




$$
\begin{aligned}
& \text { Média }=29,111 \text { expressa em p p m } \\
& \text { Desvio padrão }=45,579 \\
& \text { Erro padrão da média }=2,337
\end{aligned}
$$

Os valores que constituem a tabela I, foram divididos em quatro grupos:

\begin{tabular}{|c|c|c|c|c|}
\hline & Grupo I & Grupo II & Grupo III & Grupo IV \\
\hline $\begin{array}{l}\text { Amplitude da distribuiçãa dos va- } \\
\text { lores da dureza }(p p m) \ldots \ldots \ldots\end{array}$ & $0,8-9,2$ & $9,6-17,6$ & $18,0-33,6$ & $34,4-288,0$ \\
\hline $\begin{array}{l}\text { Amplitude da distribuição dos va- } \\
\text { lores de iôdo }(\mu \mathrm{g} / \mathrm{l}) \ldots \ldots \ldots\end{array}$ & $0,05-10,6$ & $0,00-8,46$ & $0,05-18,10$ & $0,09-21,15$ \\
\hline N.० de observações. & 96 & 94 & 96 & 95 \\
\hline Média dos valores de iôdo $(\mu \mathrm{g} / \mathrm{l})$ & 0,999 & 1,491 & 2,243 & 2,206 \\
\hline$\hat{\sigma}_{I} \quad$ Desvio padrão $\ldots \ldots$ & 2,11 & 1,69 & 3,61 & 4,21 \\
\hline$\frac{\hat{\sigma}_{I}}{\sqrt{V}}$ Êrro padrão da média & 0,215 & 0,174 & 0,368 & 0,432 \\
\hline
\end{tabular}
I, II, III e IV, cujas características se acham no quadro III.

QUADRO III

Para cada grupo foi determinada a média dos valores de iôdo $\bar{I}$, o desvio padrão $\hat{\sigma}_{1}$ e o êrro padrão da média $\frac{\hat{\sigma}_{\mathrm{I}}}{\sqrt{\mathrm{N}}}$. Testes de significancia das diferenças foram feitos $\left({ }^{*}\right)$.

Quando se observam os valores dos grupos I, II, III e IV, sucessivamente, verifica-se um aumento progressivo nas médias referentes ao iôdo, aumento êste que, entretanto, não se mantém no grupo IV. Na hipótese de não haver correlação entre dureza e iôdo, essas quatro médias não deveriam diferir significativamente entre si. Feito o teste da diferença de médias, para as médias $I_{I}, I_{I I}, I_{I I r}, e I_{I V}$, verificou-se, para alguns pares, uma diferença significante, 0 que nos levaria, nestes casos, a rejeitar a hipótese de não-correlação; todavia, o estabelecimento de uma previsão dos valores de iôdo pelos correspondentes de dureza, para aqueles casos em que a hipótese de não-correlação seria rejeitada, torna-se desprovido de qualquer significado, devido à grande transvariação existente, não só entre êsses grupos, como para todos êles.

- $\left(^{*}\right.$ Agradecemos à Docente-livre Dra. Elza S. Berquó, do Departamento de Estatística da Faculdade (Professor eatedrático: Dr. Pedro Egydio de Oliveira Carvalho), o auxílio que nos prestóu na análise estatística dos dados. 


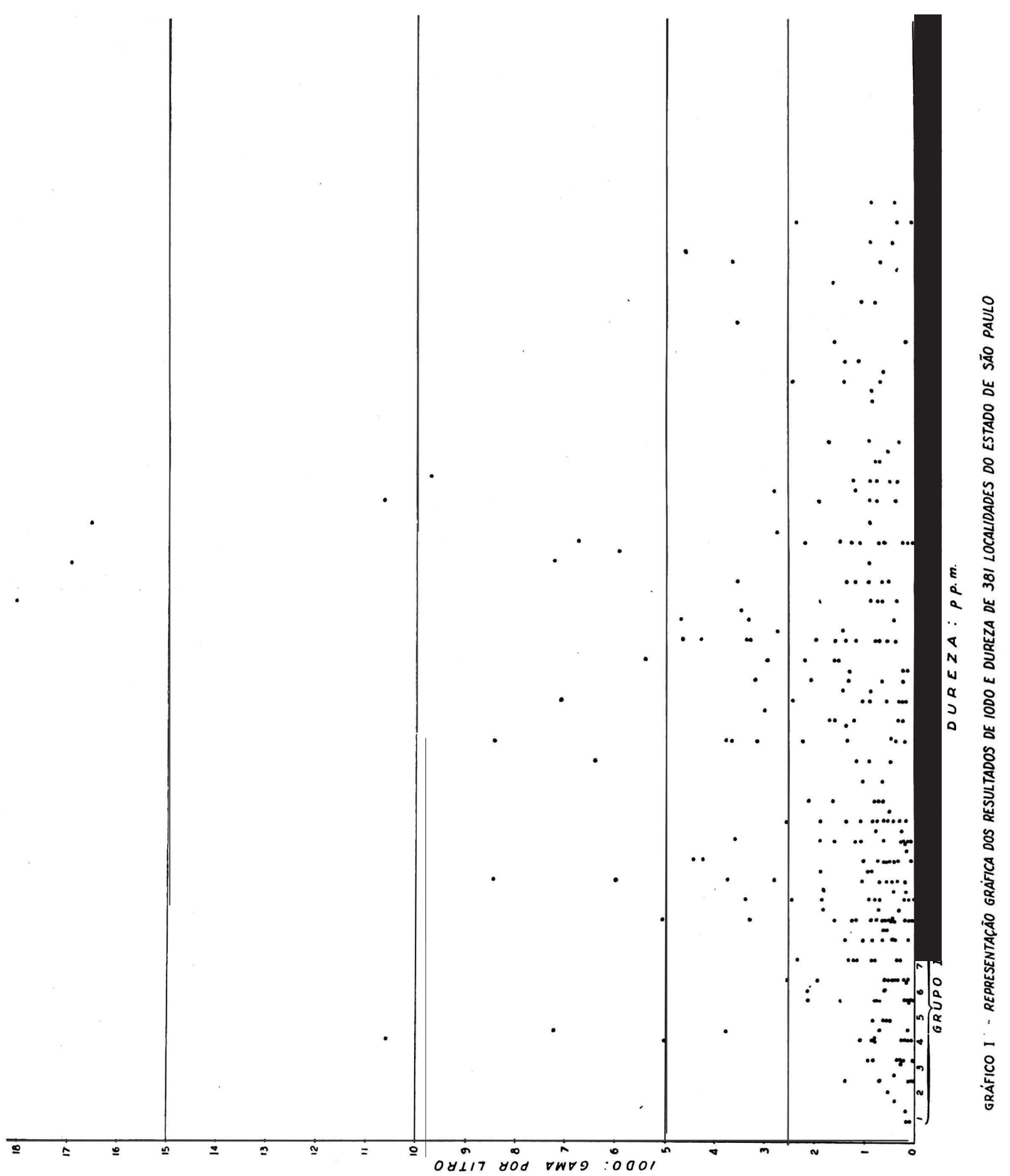


Se no grupo I encontramos taxas de iôdo que variaram desde 0,05 até $10,60 \mu \mathrm{g} /$ litro, no grupo III, embora de média superior, a variação dos resultados de iôdo $(0,05$ a $18,10 \mu \mathrm{g} /$ litro $)$ ocupou zona cujos limites abrangeram, inclusive, os do grupo I. Por outro lado, as amplitudes extremas do grupo In estão contidas dentro dos limites do grupo I.

Esta transvariação pode ser bem visualizada se observarmos o gráfico I.

Para uma mesma dureza, por exemplo, $8,8 \mathrm{p} \mathrm{pm} \mathrm{(vide} \mathrm{tabela} \mathrm{I),} \mathrm{os} \mathrm{re-}$ sultados de iôdo variaram de 0,12 a $5,05 \mu \mathrm{g} /$ litro; em 10 amostras com dureza de $10,4 \mathrm{p} \mathrm{p} \mathrm{m}$, os resultados de iôdo variaram de 0,18 , a $8,46 \mu /$ litro, e ainda em 6 amostras que apresentaram, por exemplo, dureza igual a 21,6, os resultados de iôdo foram, respectivamente, de 0,35-0,69-0,75-0,88-1,89 e 18,10 $\mu \mathrm{g} /$ /itro.

Exemplos dêstes tipos são vários e nos sugerem que, em amostras como a nossa, pode-se encontrar média de concentração de iôdo mais elevada para zonas de durezas altas, mas também nos indicam que, para uma determinada dureza, o resultado de iôdo que podemos esperar é tão variável que nos parece de nenhum valor qualquer estimativa de sua concentração pelo grau de dureza das águas.

\section{SUMÃRIO E CONCLUSÕES}

Alguns autores têm relacionado o conteúdo de iôdo das águas com o grau de dureza das mesmas. Com base nesses trabalhos, outros pesquisadores indicam a simples determinação da dureza como dado suficiente para estimar a riqueza iódica das águas.

Ao fazermos o levantamento das taxas de iôdo das águas de 381 localidades do Estado de São Paulo, resolvemos, aproveitando o mesmo material da amostra, dosar, concomitantemente, a dureza dessas águas, para pesquisar a existência ou não da possível correlação entre êsses dois dados.

Dividindo em grupos os resultados da dureza das águas, depois de dispostos em ordem crescente e determinando a média dos valores de iôdo das amostras correspondentes de cada grupo, conquanto se possa encontrar média de concentrações de iôdo mais elevada para zonas de durezas altas, a análise dos dados nos mostrou que, devido à grande transvariação existente entre êsses grupos, torna-se desprovida de valor qualquer estimativa da concentração de iôdo pelo grau de dureza das águas.

\section{SUMMARY AND CONCLUSIONS}

A number of authors have reported a relationship between iodine content and the degree of hardness of water. Based on these findings, other investigators have claimed that relative iodine content can be estimated by simply measuring the hardness. 
In our study of iodine content of waters from 381 localities in the State of São Paulo, we also measured at the same time the hardness of the samples to determine whether or not there was a correlation between the two factors.

The data were divided into groups on the basis of increasing hardness, and the mean of iodine content of each group calculated. Although a higher mean of iodine may be found in areas of high degree of hardness, the analysis of the data showed that owing to the great transvariation presented in those groups, it would be of no value to estimate the concentration of iodine by the degree of hardness of waters.

\section{REF̈ERENCIAS BIBLIOGRĀFICAS}

1. Bado, A. A. \& Trelle, R. A.: Yodo en las aguas de la Republica Argentina. An. Asoc. Quim. Arg., 17:209, 1929, cit. in Marsano 7.

2. Betz, J. D.' \& Noll, C. A.: Further studies with the direct colorimetrie hardness titration. J. Amer. Water Works Assoc., 42:749-754, 1950.

3. Cardoso, F. A.; Gandra, Y. R. \& Nazário, G.: Determinação do teor de iôdo em águas de abastecimento público de todos os municípios do Estado de São Paulo. Arq. Fac. Hig. Saúde Publ., 9:13-28, 1955.

4. Gôngora y Lopes, J.; Young, N. \& Borda, A. I.: Bocio simples y sal yodada en Colombia. Rev. Hig. (Bogotá) 24:291-330, 1950.

5. Houliham, J. E.: Total hardness of water by the versanate method. Direct titration with ethylenediaminetetraacetic acid (EDTA) Analyst, 77:158, 1952.

6. Lein, A. \& Schwartz, N.: Ceric sulfate-arsenious acid reaction in microdetermination of iodine. Analyt. Chem., 23:1507-1510, 1951.

7. Marsano, Maria Luiza A. P.: Determinacion de yodo en el agua de Mendoza. Mendoza, Universidad de Cuyo, 1952. 42p. (Boletin tecnico, n. 1).

8. Murray, M. M.; Ryle, J. A.; Simpson, B. W. \& Wilson, D. C.: Thyroid enlargement and other changes related to the mineral content of water. Med. Res. Council Memor. 18:3-39, 1948 .

9. Salas, S. M. de \& Amato, F. D.: Contribuicion al estudio de la endemia bociosa en el pais. Rev. Adm. Nac. Agua, 10:101, 1946, eit. in Marsano 7.

10. Schwarzenbach, G.; Biedermann W. \& Bangerter, F.: Complexone VI. Neue einfache Titriermethoden zur Bestimmung der Wasserhärte. Helv. Chim. Acta 29 : $811-818,1946$. 